\title{
What have we learned from
}

\section{immunotherapy? Report from the 3rd and 4th meetings of the Campania Society of Oncology Immunotherapy (SCITO)}

\author{
Paolo Antonio Ascierto ${ }^{1 *}$, Giacomo Carteni ${ }^{2}$, Cesare Gridelli ${ }^{3}$, Sandro Pignata ${ }^{4}$, Antonio Pinto ${ }^{5}$, Carmen Criscitiello ${ }^{6}$, \\ Luigi Buonaguro ${ }^{7}$, Stefano Pepe ${ }^{8}$, Roberto Mabilia ${ }^{9}$, Vincenzo Montesarchio ${ }^{10}$, Bruno Daniele ${ }^{11}$ and Sabino De Placido ${ }^{12}$
}

\begin{abstract}
Treatment strategies that target the immune system provide the opportunity for antitumor activity across multiple cancer types, regardless of mutational status or tumor histology. While many of the initial advances in immunotherapy have been in melanoma, the focus has now broadened to include many other solid as well as hematological cancers. Different immunotherapeutic approaches are being evaluated across tumor types and their various novel mechanisms of action and safety profiles offer the potential for a variety of combination regimens. Ongoing and planned investigation of these immunotherapies, alone and in combination, represents the start of a new chapter in our treatment of cancer and offers the hope of better outcomes for patients with a wide range of cancers. Recent advances in the use of immune-based approaches to treat non-small-cell lung cancer, breast cancer, ovarian cancer, gastrointestinal cancer, hepatocellular carcinoma, head and neck cancer and lymphoma were discussed at the 2015 Spring and Winter meetings of the Campania Society of Oncology Immunotherapy (SCITO) and are reported here.
\end{abstract}

Keywords: Non-small-cell lung cancer, Breast cancer, Ovarian cancer, Gastrointestinal cancer, Hepatocellular carcinoma, head and neck cancer, Hodgkin's lymphoma, Immunotherapy, Tumor-infiltrating lymphocytes

\section{Background}

Both innate and adaptive $\mathrm{T}$ cell-mediated immunity arms are believed to play coordinated roles in cancer immune surveillance. $T$ lymphocytes have a critical role in cancer development, with density of tumor-infiltrating T lymphocytes (TILs) being prognostic for improved outcomes in various cancers, although evidence concerning their antigen specificity and protective mechanisms of action is limited $[1,2]$. A conceptual model in which an adaptive $\mathrm{T}$ cell response composed of both cytotoxic CD8+ T cells (CTLs) and CD4+ Th1 cells control cancer progression has been postulated, involving cytokine production and the expansion and activation of cytotoxic CD8+ $\mathrm{T}$ cells [3].

\footnotetext{
* Correspondence: paolo.ascierto@gmail.com

${ }^{1}$ Istituto Nazionale Tumori Fondazione G. Pascale - Unit of Melanoma, Cancer Immunotherapy and Innovative Therapy, Via Mariano Semmola, 80131 Naples, Italy

Full list of author information is available at the end of the article
}

Immunotherapeutic approaches in cancer have mostly attempted to harness this adaptive $\mathrm{T}$ cell response.

The immune system, and in particular T lymphocyte activity, is regulated by a balance of co-stimulatory and co-inhibitory signals known as immune checkpoints. Normally, these act to prevent autoimmunity and damage to peripheral tissues during immune responses to infection. However, during tumour development, cancer cells can utilize immune checkpoint proteins to suppress and evade immune attack, resulting in unchecked tumor progression. Cytotoxic T-lymphocyte-associated protein-4 (CTLA-4) and programmed cell death protein 1 (PD-1) are checkpoint molecules that down-regulate T-cell activation pathways, thereby promoting tumour growth and proliferation. Inhibition of CTLA- 4 and PD- 1 binding to their ligands enhances T-cell activation and proliferation, leading to tumor infiltration by T-cells and tumor regression [4]. 
Recent advances in the use of immune-based approaches to treat cancer have led to an increased effort to assess their potential across a wider range of solid and hematological tumor types, including those for which current treatment options are limited. Developments in the use of immunotherapies in several cancers were discussed at the 2015 Spring and Winter meetings of the Campania Society of Oncology Immunotherapy (SCITO) and are reported here.

\section{Non-small-cell lung cancer}

Increased understanding of immune evasion strategies has resulted in the development of novel immunotherapies, including several agents that target PD-1 pathway. Both anti-PD-1 and PD-L1 compounds are currently in active clinical development for the treatment of non-small-cell lung cancer (NSCLC) (Table 1).

Anti-PD-1 agents include pembrolizumab and nivolumab, both of which have been approved by the US Food and Drug Administration (FDA) for the treatment of squamous or non-squamous NSCLC. As part of the KEYNOTE-001 trial, 495 patients with previously treated and treatment-naïve advanced NSCLC were treated with pembrolizumab $2 \mathrm{mg} / \mathrm{kg}$ every three weeks or $10 \mathrm{mg} / \mathrm{kg}$ every two weeks with a median duration of follow-up of 10.9 months [5]. Overall response rate (ORR) was $19.4 \%$ and median duration of response (DOR) was 12.5 months. Median progression-free survival (PFS) was 3.7 months and median overall survival (OS) was 12.0 months. In 204 patients evaluable by an immunohistochemistry (IHC) clinical trial assay, ORR was $45.2 \%$ in those with membranous PD-L1 expression in $\geq 50 \%$ of tumor cells (proportion score [PS] $\geq 50 \%$ ), compared to $16.5 \%$ in patients with PS 1-49 and $10.7 \%$ in patients with PS $<1 \%$. Among patients with a PS $\geq 50$, median PFS was 6.3 months; median OS was not reached. The relationship between ORR and PD-L1 expression was observed in both previously treated and treatment-naïve patients. Pembrolizumab is being further investigated versus docetaxel in pretreated NSCLC patients with PD-L1 $\geq 1 \%$, showing increased effectiveness both in terms of ORR and OS in patients with PD-L1 $\geq 50$ \% (KEYNOTE-010; NCT01905657) [6], versus standard of care in treatment-naïve NSCLC patients with PD-L1 $\geq 50$ \% (KEYNOTE-024; NCT02142738) and versus placebo in early-stage NSCLC after resection and standard adjuvant therapy (PEARLS; KEYNOTE-091; NCT02504372).

Nivolumab has also been shown to be effective and well tolerated in patients with non-squamous NSCLC in phase II/III studies [7, 8]. In addition, in the CheckMate 012 multi-arm phase I study of nivolumab, platinum doublet plus nivolumab was promising with an ORR of 33-50\%, 24-week PFS of 36-71 \% and 1-year OS of 59-87 \% [9]. Similarly, nivolumab was effective compared with docetaxel in patients with advanced non-squamous NSCLC who had progressed during or after platinumbased doublet chemotherapy [10]; median OS was 12.2 months with nivolumab $(n=292)$ versus 9.4 months with docetaxel $(n=290)$ and the 1 -year OS rate was $51 \%$ versus $39 \%$. ORR was $19 \%$ with nivolumab versus $12 \%$ with docetaxel and median DOR was 17.2 versus 5.6 months. Almost one-quarter (24\%) of patients on nivolumab were treated beyond RECIST v1.1-defined progression and a non-conventional benefit was observed in 16 patients (not included in best overall response). Nivolumab was associated with greater efficacy than docetaxel across all endpoints in subgroups defined according to pre-specified levels of tumor-membrane PD-1 expression ( $\geq 1 \%, \geq 5 \%$, and $\geq 10 \%$ ). Nivolumab has also shown improved efficacy versus docetaxel in 272 patients with advanced squamous-cell NSCLC [11]. Median OS was 9.2 months with nivolumab versus 6.0 months with docetaxel; risk of death was $41 \%$ lower with nivolumab (hazard ratio [HR] 0.59; $95 \%$ CI: 0.44-0.79; $p<0.001$ ). At 1 year, the OS rate was $42 \%$ with nivolumab versus $24 \%$ with docetaxel. ORR was independent of PD-L1 expression and consistently higher for nivolumab versus docetaxel.

The anti-PD-L1 agent durvalumab (MEDI4736) has also shown encouraging antitumor activity and a manageable safety profile in patients with NSCLC. In a phase I trial of patients with various solid tumor types, disease control rate (DCR) at 12 weeks was $41 \%$ and ORR was $16 \%$ among 162 evaluable patients with NSCLC, with activity observed in both squamous and non-squamous histologies [12]. ORR was higher in PD-L1+ than PD-L1- patients (25\% versus $10 \%)$. Durvalumab is now being assessed as sequential therapy in patients with locally advanced unresectable stage III NSCLC who have not progressed following platinum-based chemoradiation therapy (PACIFIC trial, NCT02125461). Another PD-L1 inhibitor in development is atezolizumab (MPDL3280A). In a phase I dose-escalation and expansion study involving 88 patients, ORR was $21 \%$ and 24-week PFS rate was $42 \%$ [13]. PD-L1 expression appeared to be predictive for clinical benefit. No maximum tolerated dose, dose-limiting toxicities or treatment-related deaths were observed, with the majority of adverse events (AEs) grade $1-2$. In interim analysis from the randomized, phase II POPLAR trial of previously treated NSCLC patients, median OS was 11.4 months with atezolizumab versus 9.5 months with docetaxel (HR 0.78). Improved efficacy was observed with increasing PD-L1 expression; patients with the lowest PDL1 did not appear to benefit from atezolizumab treatment [14]. Fewer patients receiving atezolizumab experienced grade $\geq 3$ AEs and there were no unexpected toxicities. Atezolizumab is being further assessed in PD-L1+ locally advanced or metastatic NSCLC patients (BIRCH phase II 
Table 1 Overview of PD-L1 and PD-1 inhibitors currently in development

\begin{tabular}{|c|c|c|c|c|}
\hline Compound & Lead company & Antibody type & Affinity $/ K_{2}$ & Selected tumour types assessed \\
\hline \multicolumn{5}{|l|}{ Anti-PD-L1 } \\
\hline Atezolizumab (MPDL3280A) & Roche & $\begin{array}{l}\text { Engineered lgG1 } \\
\text { (no ADCC) }\end{array}$ & $0.4 \mathrm{nM}$ & $\begin{array}{l}\text { NSCLC: ORR: } 21 \% \text {; } 24-\text { week PFS rate: } 42 \% \\
\text { Median OS: } 11.4 \text { months (vs } 9.5 \text { with } \\
\text { docetaxel) [10] }\end{array}$ \\
\hline Durvalumab (MEDI4736) & AstraZeneca & Modified IgG1 (no ADCC) & NA & $\begin{array}{l}\text { NSCLC: } 12 \text {-week DCR: } 41 \% \text {; ORR: } 16 \% \text { [8] } \\
\text { HNSCC: ORR: } 12 \% \text { (25\% in PD-L1+ patier }\end{array}$ \\
\hline
\end{tabular}

HNSCC: ORR: $12 \%$ (25\% in PD-L1+ patients), 24-week DCR: $16 \%$ (25\% in PD-L1+ patients) [43]

Currently being assessed in first-line recurrent or metastatic HNSCC in combination with tremelimumab [44].

Also being assessed as monotherapy or in combination with tremelimumab in bladder, gastric, pancreatic, HCC and blood cancers.

BMS-936559

Anti-PD-1

Nivolumab
Bristol-Myers Squibb

$\operatorname{lgG} 4$ (humanised)

Bristol-Myers Squibb

$\operatorname{lgG} 4$
$2.6 \mathrm{nM}$

Approved in previously-treated advanced squamous or non-squamous NSCLC and RCC, metastatic melanoma, and $\mathrm{HL}$ that has relapsed or progressed after HSCT and post-transplantation brentuximab vedotin. Platinum-resistant ovarian cancer: ORR: $23 \%$; DCR: $54 \%$ [25].

Advanced HCC: CR: 2/39 (5 \%); PR 7/39 (18\%); 6-month OS: $72 \%$ [32]

Heavily pretreated RR-HL: ORR: $87 \%$; CR: $22 \%$. Mmedian DOR and median PFS not yet reached at median follow up of 101 weeks [53].

RR-DLBCL: ORR: $36 \%$; median overall DOR of 22 weeks [62].

RR-FCL: ORR: $40 \%$. At a median follow up of 91 weeks, the median DOR for responding patients was not yet reached [62].

Approved in previously-treated advanced
Biomarker status

PD-L1 tested with SP142 mAb clone, with an automated system, and evaluated on both tumoral and immune-infiltrate cells, with a cut-off of $\geq 5 \%$ and $\geq 10 \%$ Biomarker status still experimental

PD-L1 tested with SP263 mAb clone, with an automated system, on tumoral cells, with a cut-off of $\geq 25 \%$

Biomarker status still experimental

PD-L1 tested with 28-8 mAb clone, with an automated system, on tumoral cells, with a cut-off of $\geq 5 \%$

Biomarker status still experimental

PD-L1 tested with 28-8 mAb clone, with an automated system, on tumoral cells, with a cut-off of $\geq 5 \%$

Biomarker status: No testing required squamous or non-squamous NSCLC and metastatic melanoma.

Ovarian cancer: ORR: $11.5 \%, 23.1 \%$ of patients had evidence of tumor reduction; DCR: $34.6 \%$ [27].
PD-L1 tested with 22C3 mAb clone, with an automated system, on tumoral cells, with a cut-off of $\geq 1 \%$ and $\geq 50 \%$ (strong positive). Biomarker status testing required (US) 
Table 1 Overview of PD-L1 and PD-1 inhibitors currently in development (Continued)

Esophageal cancer: ORR : $23 \%(n=5)$; best response was SD in $18 \%(n=4)$ and PD in $59 \%(n=13)$ [30].

Advanced gastric cancer: ORR: $22 \%(95 \% \mathrm{Cl}$

$10-39)$ by central review and $33 \%(95 \% \mathrm{Cl}$ :

19-50) by investigator review. Median time to

response was 8 weeks (range 7-16); median

DOR: 24 weeks; 6-month PFS: $24 \%$ and

6-month OS: $69 \%$ [31].

HNSCC: ORR (confirmed and unconfirmed):

$18.2 \%(95 \%$ Cl: 11.1-27.2) with 18 partial

responses and 31 with SD [39\}.

Recurrent/metastatic nasopharyngeal

carcinoma: CR: 1/27; PR: 6/27; SD 14/27; The

best ORR (confirmed and unconfirmed):

$25.9 \%(95 \% \mathrm{Cl}, 11.1-46.3)[42]$.

RR-HL: ORR: $65 \%$; CR: $16 \%$; median PFS at

24 weeks: $69 \%$ with a median DOR

$\geq 24$ weeks in $71 \%$ of patients who achieved

complete or partial response [54, 55].

Being assessed in metastatic colorectal cancer

in combination with stereotactic body

radiation therapy.

Other $^{\mathrm{a}}$

Pidilizumab

Medivation

$\lg$ G1 (humanised)

NA

Relapsed/refractory DLBCL: CR:34 \%; ORR:

$51 \%$ in patients with measurable disease

after transplant. In the whole cohort of

patients, 16-month PFS from first treatment:

$72 \%$; OS > $80 \%$ [60]

Relapsed/refractory FCL in combination with

rituximab: ORR: 66 \%; CR: 52 \%; Median PFS

for all patients:18.8 months, and not reached,

at time of analysis, for the 19 patients with

CR/PR

$A D C C$ antibody-dependent cell-mediated cytotoxicity, $C R$ complete response, $D C R$ disease control rate, $D L B C L$ diffuse large $B$ cell lymphoma, DOR duration of response, $F C L$ follicular lymphoma, $H C C$ hepatocellular carcinoma, HL Hodgkin's lymphoma, HNSCC head and neck squamous cell carcinoma, HSCT hematopoietic stem cell transplantation, mAb monoclonal antibody, NA not available, NSCLC non-small cell lung cancer, ORR overall response rate, OS orall survival, PD prd

aPidilizumab was initially believed to have been an anti-PD-1 agent; however, it has recently been stated by Medivation that this is not the case although its exact mechanism of action has not been revealed 
study; NCT02031458) and versus docetaxel as second- or third-line treatment in locally advanced or metastatic NSCLC patients (OAK phase III study; NCT02008227).

\section{Breast cancer}

TILs have been consistently documented in breast cancer (BC) and have been associated with prognosis. In a seminal paper published in 1992, Aaltoma and colleagues reported that lymphocytic infiltration was associated with a good prognosis, but only among rapidly proliferating tumors [15]. Similarly, the presence of TILs is observed in some $\mathrm{BCs}$ and has been reported to be a potential prognostic and predictive marker in some disease types, especially triple-negative (TNBC) and HER2+ BC $[16,17]$.

Retrospective studies from (neo)-adjuvant trials have evaluated whether TILs could identify patients with a specific outcome. Denkert et al. have shown that TIL+ tumors present a good outcome as opposed to their negative counterparts $[18,19]$. Based on this observation, the ability of TILs to quantify the residual risk of relapse after adjuvant treatment has been evaluated in two retrospective analyses from randomized trials $[17,20]$. More recently, the association between TILs and pathological complete response (pCR) has been further evaluated by Denkert and colleagues [19, 21]. In the GeparSixto trial, increased pCR rates were observed in TNBC and HER2+ BC with high-TIL, compared with low-TIL tumors [19]. In the NeoALTTO trial that randomized 455 women with HER2 + early-stage $\mathrm{BC}$ to receive either trastuzumab or lapatinib or both agents followed by the addition of weekly paclitaxel and three cycles of FEC after surgery, the presence of TILs at diagnosis was an independent prognostic marker for pCR and event-free survival [21].

In a retrospective study of 304 patients with TNBC and residual disease after neoadjuvant chemotherapy, both intratumoral and stromal TILs at surgery were associated with better prognosis, especially in patients with large tumor burden (Fig. 1) [22]. Interestingly, in this study $85 \%$ of the samples with high-TIL after neoadjuvant chemotherapy were low-TIL on core biopsies preneoadjuvant chemotherapy. Also, the prognostic value of TILs in TNBC has been recently confirmed by two phase III adjuvant trials [23].

Moreover, in a pooled analysis of six clinical trials involving 991 patients with TNBC treated with anthracyclinebased chemotherapy, each $10 \%$ increase in stromal TILs was associated with a $14 \%$ relative reduction in invasive disease-free survival (IDFS) events (HR $=0.86,95 \% 0.80$ $0.93, p<0.0001)$ and a $17 \%$ relative reduction in deaths $(\mathrm{HR}=0.83,95 \% \mathrm{CI} 0.76-0.91, p=0.0001)$ [24]. In a multivariate analysis adjusted for age, nodal status, tumor size and chemotherapy regimen, the HR for each $10 \%$ increase in stromal lymphocytic infiltration was $0.86(0.76-0.92)$ for IDFS events and 0.84 (0.76-0.92) for death. Moreover, stromal TILs added significant independent prognostic information for both IDFS (chi2 $=17.9 ; p<0.0001$ and OS (chi2 = 16.7; $p<0.0001$ ).

TILs may also be predictive of a better response to treatment. In the BIG 02-98 trial, only in the HER2positive $\mathrm{BC}$ subgroup was there evidence of a heterogeneous treatment response according to the percentage of TILs. In this subgroup, patients with high-TIL showed a higher benefit to an immunogenic chemotherapy, such
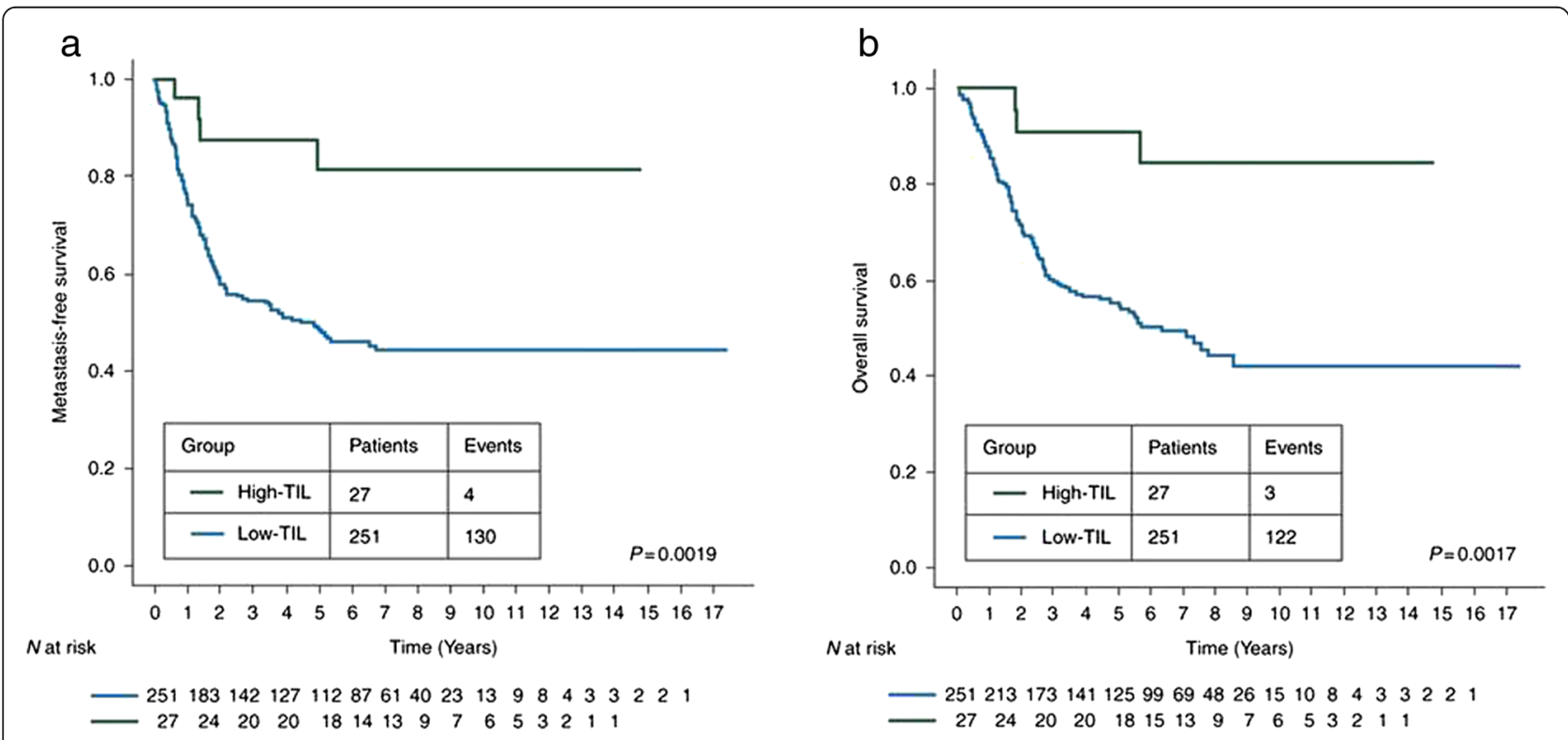

Fig. 1 Prognostic value of tumor-infiltrating lymphocytes on residual disease after neo-adjuvant chemotherapy for triple-negative breast cancer. [22] Estimated Kaplan-Meyer curves of metastasis-free survival a and overall survival $\mathbf{b}$ for all patients 
as anthracyclines, as compared to those with low-TIL tumors [20].

In the FinHER trial, 232 patients with HER2+ disease were randomized to nine weeks of chemotherapy with or without trastuzumab [17]. Each $10 \%$ increase in TILs was significantly associated with decreased distant recurrence in patients receiving trastuzumab. However, exploratory analyses from a subset of HER2+ patients in the N9831 study indicated that stromal TILs were associated with improved recurrence-free survival in patients treated with chemotherapy alone but not in patients treated with chemotherapy plus trastuzumab [25].

Evidence that a positive TIL status is associated with better outcome supports the development of immunotherapeutic strategies in patients with BC. In addition, evaluation of lymphocytic infiltrate status could help identify a subset of TIL- patients with TNBC or HER2+ $\mathrm{BC}$ who warrant additional therapy. Also, the ability to convert TIL- tumors into TIL+ tumors needs further investigation; such an approach might allow selecting which patients with TIL- TNBC are candidates for novel therapies in the (neo)-adjuvant setting.

\section{Ovarian cancer}

Despite advances in combination chemotherapy regimens, current options for ovarian cancer patients are inadequate and the majority of patients will relapse. An important factor in the poor outcomes seen in patients with ovarian cancer is the lack of effective second-line treatments. As such, novel therapies need to be integrated into treatment strategies to achieve durable clinical outcomes. Immunotherapy offers a novel and promising therapeutic strategy.

Evidence for an immune role in ovarian cancer is shown by the observation that the presence of $\mathrm{CD} 3+$ and $\mathrm{CD} 8+$ TILs is associated with favorable OS in ovarian cancer. In addition, several tumor-associated antigens (TAAs) that are up-regulated in tumor tissue and ascites of ovarian cancer patients have been identified (e.g., Her2/neu, folate receptor $\alpha, \mathrm{p} 53$, CA125, and members of the cancer-testis antigen family such as MAGE -A4 and NY-ESO-1). Potential immunotherapeutic approaches in ovarian cancer include monoclonal antibodies, immune checkpoint inhibitors, vaccines and adoptive cell therapy (ACT).

Antibodies include catumaxomab, a trifunctional bispecific (anti-EpCAM/anti-CD3) antibody that has been shown to improve puncture-free interval in heavily pretreated patients with chemotherapy-refractory ovarian cancer and recurrent symptomatic malignant ascites [26], the anti-EGFR agents cetuximab and panitumumab [27, 28], and antibodies that target tumor-associated macrophage (e.g., anti-CCL22, anti-B7-H4, anti-CSF-1R).

Checkpoint inhibitors are also being investigated in ovarian cancer. PD-L1 expression on monocytes in the ascites and blood of patients with ovarian cancer correlates with poor clinical outcome and cytotoxicity assays have revealed that PD-L1 overexpression on murine ovarian cancer cells inhibits cytotoxic $\mathrm{T}$ lymphocyte (CTL) degranulation and reduces CTL-mediated tumor lysis. In the first trial of nivolumab in patients with platinum-resistant ovarian cancer $(n=15)$, treatment resulted in a response rate of $23 \%$ and DCR of $54 \%$ [29]. In follow-up of two patients with complete response and one with a partial response, antitumor responses were durable and continued after discontinuation of nivolumab [30]. Pembrolizumab is also being assessed in ovarian cancer. In the ongoing non-randomized, phase Ib KEYNOTE-028 trial in patients with PD-L1+ solid tumors, pembrolizumab showed antitumor activity in an interim analysis of the patient cohort with heavily pretreated metastatic ovarian cancer $(n=26)$; ORR was $11.5 \%$, $23.1 \%$ of patients had evidence of tumor reduction and DCR was $34.6 \%$ [31]. Treatment was also generally well tolerated with no discontinuations due to toxicities. The anti-PD-L1 antibody, avelumab (MSB0010718C), has also shown promising results in ovarian cancer. In a phase $\mathrm{Ib}$, open-label expansion trial, four of 23 patients (17.4 \%) followed-up for $\geq 2$ months achieved an partial response, 11 (47.8\%) had stable disease, and two had $>30 \%$ tumor shrinkage after progression was reported [32]. Median PFS was 11.9 weeks and PFS rate at 24 weeks was $33.3 \%$ (95\% CI 11.5-57.2). The safety profile was acceptable, with fatigue, nausea, and diarrhea the most commonly reported drug-related AEs, and phase III studies are ready to start in first-line and platinum-sensitive recurrence.

Another possible approach is targeting the indoleamine 2,3-dioxygenase (IDO) pathway, an important mechanism of tumor-related immunosuppression. In ovarian cancer, IDO expression is prevalent in $>50 \%$ of surgically resected tissue, correlates with a reduced number of CD8+ TILs and NK cells, promotes tumor angiogenesis and is positively associated with impaired survival in serous-type disease. The first IDO-targeted therapy is 1-methyl-tryptophan (1-MT), a small molecule inhibitor of IDO, that prolonged survival when added to paclitaxel in an IDO-overexpressing murine ovarian cancer model and that is being evaluated in phase I clinical trials in different solid tumors with encouraging results.

Vaccine strategies in ovarian cancer include Cvac, an $e x$ vivo dendritic cell vaccine that has shown promise in a phase II study in 63 patients in second remission [33], an in vivo dendritic cell vaccine based on the MSLN-Hsp70 fusion protein, peptide vaccines, and recombinant viral vaccines that utilize genetically modified viruses as vectors for introducing TAA-encoding DNA into cells within the body e.g., PANVAC-VF. Finally, ACT, a process that involves using autologous or allogeneic antitumor 
lymphocytes to induce cancer regression may have a role with the activation of endogenous T-cell immunity having been induced to enhance the elimination of tumor cells and the development of tumor-specific memory responses in a mouse model of ovarian cancer. Multiple ovarianspecific tumor antigens are being used in chimeric antigen receptor (CAR) development and ACT strategies are moving towards the clinic.

As in other cancers, the use of combination immunotherapeutic strategies is likely to be important in ovarian cancer. Strategies being investigated include anti-CTLA-4 and GVAX, a granulocyte-macrophage colony-stimulating factor (GM-CSF) gene-transfected tumor cell vaccine and nivolumab and an IDO inhibitor (INCB24360). Identification of the optimal treatment combinations should translate to substantial improvements in long-term clinical benefit.

\section{Gastrointestinal cancer}

Despite some recent improvements, the prognosis for advanced gastric and esophageal cancer remains poor, with limited treatment options in the metastatic or unresectable setting and a typical median survival of less than one year. As with many other cancer types, immune checkpoint inhibitors may provide a new therapeutic option in the treatment of gastrointestinal (GI) neoplasms.

PD-L1 is frequently overexpressed in esophageal cancer and may be associated with a poor prognosis. In a preliminary analysis of the non-randomized, phase Ib KEYNOTE-028 trial of pembrolizumab for PD-L1+ advanced solid tumors, 90 patients with esophageal cancer were screened of whom 37 (41 \%) had PD-L1+ tumors [34]. In the 23 patients treated to date, ORR (confirmed and unconfirmed) was $23 \%(n=5)$; the best response was stable disease in $18 \%(n=4)$ and progressive disease in $59 \%(n=13)$. Six patients $(26 \%)$ experienced drugrelated AEs, including two (9\%) with grade 3 events. There were no grade 4 events and no patients died or discontinued due to an AE.

In the KEYNOTE-012 trial, the safety and efficacy of pembrolizumab was assessed in patients with PD-L1+ advanced gastric cancer [35]. Of the 162 patients screened, 65 (40\%) were PD-L1+ and 39 were treated with a median follow-up of 8.8 months (range 6.2-12.6). ORR was $22 \%$ (95\% CI: 10-39) by central review and 33 \% (95 \% CI: 19-50) by investigator review. Median time to response was 8 weeks (range 7-16), with a median DOR of 24 weeks. The 6-month PFS rate was $24 \%$ and 6-month OS was $69 \%$. PD-L1 expression level was associated with ORR. Treatment was well tolerated with four patients experiencing grade 3/4 drug-related AEs. Pembrolizumab is being further evaluated alone or in combination with chemotherapy in the phase II KEYNOTE-059 study of patients with recurrent or metastatic gastric or gastroesophageal junction adenocarcinoma (NCT023354110) and as second-line therapy in the phase III KEYNOTE-061 study versus paclitaxel in patients with advanced gastric or gastroesophageal junction adenocarcinoma after progression on chemotherapy (NCT02370498).

\section{Hepatocellular carcinoma}

The prognosis for hepatocellular carcinoma (HCC) is generally poor given the low effectiveness of available treatments and the overall 5-year survival rate is approximately 5-6\%. Immunotherapeutic interventions may represent a novel and effective approach, although only few immunotherapy trials for HCC have been conducted and results to date have been modest.

Nivolumab was assessed in a phase I/II trial in patients with advanced hepatocellular carcinoma [36]. Drugrelated AEs of any grade occurred in 29 of 41 patients (71 \%); $17 \%$ of patients had grade 3/4 AEs. A doselimiting toxicity occurred in an uninfected patient receiving nivolumab $10 \mathrm{mg} / \mathrm{kg}$ but no maximum tolerated dose was defined. Response was evaluable in 39 patients; two had a complete response (5\%) and seven had partial responses (18\%); responses were durable. OS rate at 6 months was $72 \%$.

Another potential option for $\mathrm{HCC}$ is the development of vaccines, including pulsed dendritic cell-based vaccines and peptide vaccines. The first $\mathrm{HCC}$ vaccine tested was based on CD8+ T-cell epitopes specific for $\alpha$-fetoprotein (AFP), showing the generation of AFP-specific T-cell responses in vaccinated subjects [37]. The same group also conducted a subsequent phase I/II trial administering AFP epitopes presented by autologous dendritic cells loaded ex vivo; however, this only produced a transient CD8+ T-cell responses, possibly caused by the lack of CD4+ support [38]. To increase the number of tumor TAAs elicited by the vaccine, approaches using autologous dendritic cells pulsed ex vivo with a lysate of the autologous tumor [39] or of hepatoblastoma cell line HepG2 [40] have been evaluated in clinical trials. However, results have been unsatisfactory.

One explanation for the limited success of $\mathrm{HCC}$ vaccines to date is that the liver is an inherently immunosuppressive microenvironment, a state that may be further exacerbated by chronic inflammation due to hepatitis infection (Fig. 2). Moreover, TAAs used in such clinical trials are not $\mathrm{HCC}$-specific. In order to improve the efficacy of HCC vaccines, new and more specific TAAs and/or tumor epitopes need to be identified, both HLA class I and II restricted, aiming at inducing CD4+ as well as $\mathrm{CD} 8+$ activation. In addition, improved immune responses elicited by $\mathrm{HCC}$ vaccines may be achieved by adjuvant strategies that increase the immunogenicity of the vaccine antigen and/or counteract 


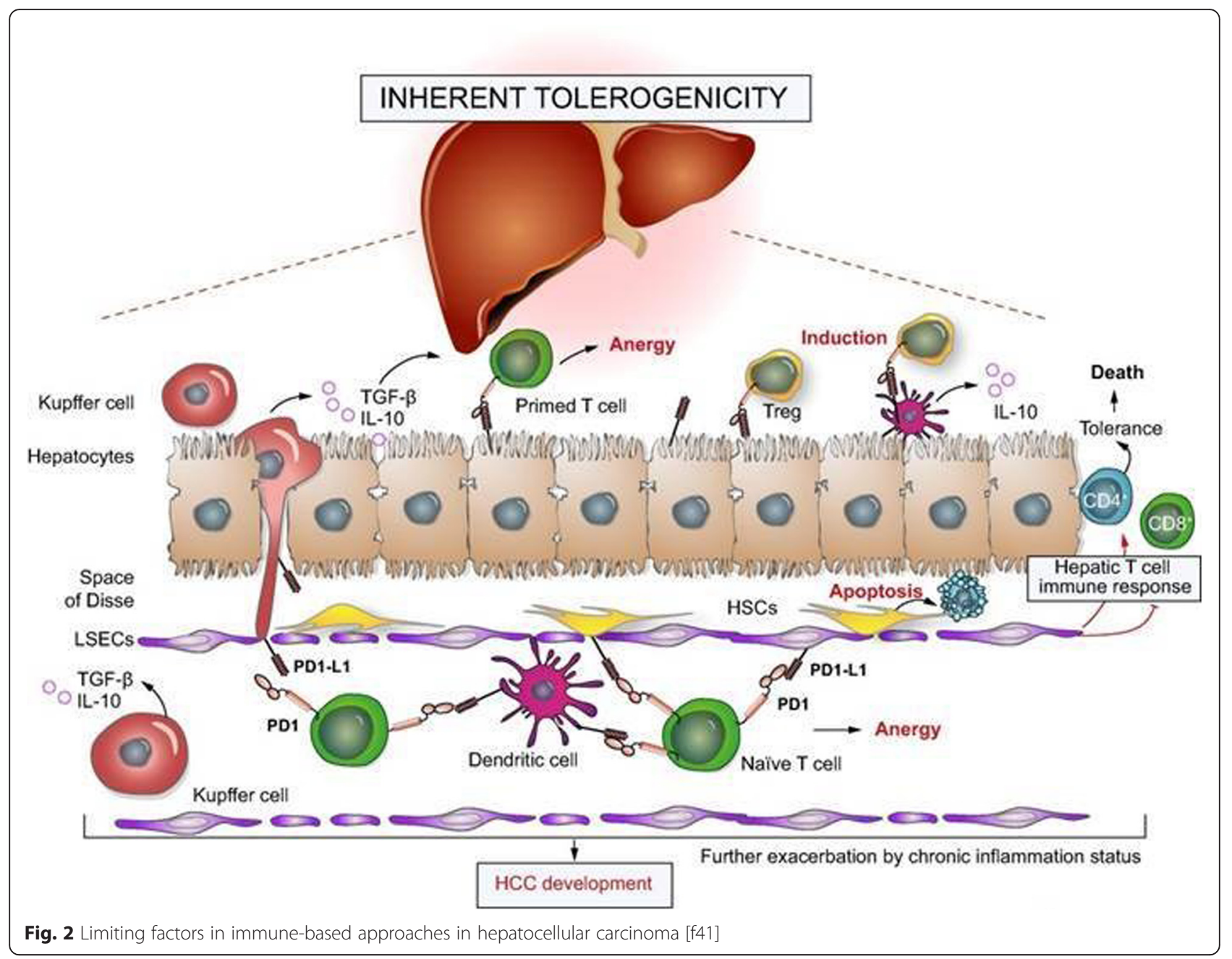

the immunosuppressive tumor environment. These include combination approaches with chemotherapy or loco-regional treatments (e.g., tumor ablation, transarterial chemoembolization [TACE]), the use of novel immunomodulatory adjuvants, and delivery systems that increase antigen presentation (e.g., biodegradeable nanoparticles as antigen carriers) [41].

An important initiative in the development of a vaccine for HCC is the EU-supported HEPAVAC project (www.hepavac.eu), aiming at developing a novel therapeutic HCC vaccine with multiple TAAs that are presented on the surface of primary HCC cells. This will involve on an 'off-the-shelf' vaccine comprising 18 newly identified MHC-I and II tumor-associated peptides (TUMAPs) naturally processed and presented on primary tumor tissues from HCC patients together with an actively personalised vaccine (APVAC) approach involving patient-specific mutated peptides. Both vaccines will be combined with a novel and potent RNA-based immunomodulator (RNAdjuvant ${ }^{\circ}$ ). Feasibility, safety and biological efficacy will be evaluated in a randomized, controlled multicentre phase I/II clinical trial, and will hopefully result in the first multi-epitope, multitarget and multi-HLA allele therapeutic cancer vaccine for this frequent and aggressive disease [42].

\section{Head and neck cancer}

Treatment options for recurrent/metastatic head and neck squamous cell carcinoma (HNSCC) remain poor with a median OS of 10 months in the first-line setting and 6 months in previously treated patients. Human papillomavirus (HPV) is recognized as the causative agent of HNSCC in a growing subset of patients. Prominent immune escape during malignant progression is observed in HNSCC, with the PD-1/PD-L1 pathway playing an important role. The majority of HPV+ and a subset of HPVHNSCC tumors are PD-L1+. Thus, blocking PD-1 interaction with PD-L1 or PD-L2 may reactivate immune surveillance and elicit antitumor activity.

Pembrolizumab has demonstrated antitumor activity in multiple tumor types, including HNSCC. The KEYNOTE012 phase $1 \mathrm{~b}$ multi-cohort study in patients with advanced 
solid tumors included a HNSCC expansion cohort with 132 patients irrespective of PD-L1 or HPV status who received fixed-dose pembrolizumab $200 \mathrm{mg}$ every three weeks [43]. In 99 patients available for preliminary efficacy analysis, ORR (confirmed and unconfirmed) was $18.2 \%$ (95 \% CI: 11.1-27.2) with 18 partial responses and 31 with stable disease. Pembrolizumab was active in both HPV+ and HPV- patients. Drug-related AEs of any grade occurred in $47 \%$ of patients and drug-related grade $\geq 3 \mathrm{AEs}$ occurred in $7.6 \%$. In a further evaluation of immunerelated gene expression patterns in 43 of these patients, the 'Interferon- $\gamma$ (IFN- $\gamma$ ) 0 10-gene', 'expanded-immune 28gene' and 'de novo' signatures showed significant associations with ORR and PFS.

In the expansion cohort of the phase Ib KEYNOTE028 trial, the antitumor activity of pembrolizumab $10 \mathrm{mg} / \mathrm{kg}$ every two weeks is being assessed in patients with PD-L1+ recurrent/metastatic nasopharyngeal carcinoma (NPC). PD-L1 expression in NPC is upregulated by Epstein-Barr virus (EBV) induced activation of LMP1 and IFN- $\gamma$ pathways and PD-1/PD-L1 expression may correlate with poor prognosis [44, 45]. Among 27 patients, one had a complete response, six had partial responses and 14 had stable disease. The best overall (confirmed and unconfirmed) response rate was $25.9 \%$ (95 \% CI, 11.1-46.3) [46]. Pembrolizumab was well tolerated with drug-related AEs observed in $70.4 \%$ of patients. This represents the first demonstration of antitumor activity of a PD-1 inhibitor in recurrent/metastatic NPC and further investigation is planned.

Durvalumab is also being assessed in HNSCC, in an ongoing open-label, phase I/II study in multiple solid tumor types [47]. In 51 of 62 HNSCC patients evaluable for response with $\geq 24$ weeks of follow-up, ORR was $12 \%$ (25\% in PD-L1+ patients), and DCR at 24 weeks was $16 \%$ (25\% in PD-L1+ patients). Drug-related AEs were observed in $60 \%$ of patients, with fatigue, diarrhea, and nausea the most frequent. Grade $\geq 3$ drug-related AEs were reported in $7 \%$ of patients: rash $(n=2)$, and increased GGT, fatigue, and tumor inflammation $(n=1$ for each). Durvalumab is also being assessed in combination with the CTLA-4 inhibitor tremelimumab versus standard of care for the treatment of first-line recurrent or metastatic HNSCC [48].

\section{Lymphomas}

Application of immunotherapy to the management of lymphomas poses unique challenges and opportunities since these malignancies originate from the immune system itself [49]. Lymphomas represent the fifth most common cancer in developed countries and collectively display an age-adjusted incidence of about 23 cases per 100,000 individuals. In the Western world, the greatest majority of lymphoid malignancies arise from mature B cells that, throughout the complex pathway to generate cells producing antibodies with a high specificity and avidity, accumulate genetic and epigenetic changes incompatible with their proper function. Studies have shown that most B cell non-Hodgkin lymphomas (NHL) and Hodgkin lymphoma (HL) derive from these nonfunctional B cells escaping the apoptotic death within secondary lymphoid tissues [50]. Similar mechanisms, involving errors in the generation of a functionally active T-cell receptor have been implied in the pathogenesis of the more rare T-cell NHLs [50]. The cellular and clinical heterogeneity of lymphomas reflects the complexity of the human immune system including the intricate patterns of cellular interplay underlying the functional regulation of the immune response. Beyond the focus on the genetic changes and cell-signaling aberrations that may act as targets for a tailored therapeutic intervention across the various lymphoma subtypes, studies have recently recognized that mechanisms operated by lymphoma cells to evade antitumor immunity through the generation and maintenance of a tolerogenic tumor microenvironment can represent a further area to build newer immunotherapeutic approaches [51, 52].

Among these strategies, immune checkpoint inhibition is emerging with considerable promise in the treatment of NHL and HL. However, at variance with solid tumors, lymphomas are characterized by a more promiscuous pattern of reciprocal expression of the receptor-ligand pairs of the PD-1 pathway among tumor cells and nonmalignant lymphoid cells. These expression patterns are also significantly variable across the specific lymphoma subtypes [53]. The expression of PD-L1/2 on tumor cells can be regulated by IFN- $\gamma$, IL- 4 and other cytokines produced in the tumor environment typical of some lymphomas, while, in other lymphoma subtypes, the constitutive expression of PD-L1/2 on tumor cells is related to a specific acquired genetic trait. Tumor cells of some lymphoma subtypes do not directly express PD-1-L, but PD-L1+ histiocytes and other microenvironmental cells are present which contribute to the exhaustion of tumor PD-1+ infiltrating T/NK cells. As an example, while in HL and some biologically related NHL, such as the primary mediastinal B-cell lymphoma (PMBCL), tumor cells clearly overexpress PD-L1/2, in follicular cell lymphoma (FCL), the prototype of indolent NHL, malignant cells are usually negative for expression of both PD-1 ligands. In aggressive NHL, the patterns of expression of PD-1 and its ligands in tumor cells might not differ from their non-malignant counterpart cells or display a subtype-divergent picture as in the case of the germinal-center (GC) and activated B cell $(\mathrm{ABC})$ variants of diffuse large B-cell lymphoma (DLBCL). Such heterogeneity depends on specific genetic features acquired by tumor cells, the variable milieu of cytokinemediated and cell contact-dependent interactions among 
malignant and non-malignant cell populations within the lymphoma microenvironment or on the combination of all these factors.

\section{Hodgkin's lymphoma}

PD-L1 and/or PD-L2 over-expression on lymphoma cells may play a critical role in immune evasion in patients with HL. The PD-L1 and PD-L2 genes are located on chromosome 9p24.1, over-amplification of which represents a specific structural alteration that occurs in some cases of HL; this increases the gene dosage of PD-1 ligands as well as their induction by JAK2 whose gene is located in the same amplified chromosomal trait [54]. As such, the PD-1 pathway and JAK2 may represent complementary therapeutic targets in HL (Fig. 3). However, tumor cells from HL cases with normal 9p24.1 copy numbers also appear to over express PD-1 ligands. The presence of an AP-1-responsive enhancer in the PD-L1 gene along with the constitutive AP-1 activation, typical of tumor cells of HL, may then depict an alternative mechanism leading to $\mathrm{PD}-1$ ligand overexpression in this lymphoma [55]. Finally, the demonstration that the EBV-encoded latent membrane protein 1 increases PD-L1 promoter activity supports a third mechanism for PD-L1 overexpression in HL, since EBV-encoded proteins can be found in about $40 \%$ of HL cases [55]. Such concurrency of mechanisms may explain why overexpression of PD1-ligands is a phenotypic trait common to almost all cases of HL. While this situation clearly accounts, from one side, for the impressive clinical efficacy of PD1-inhibitors in HL, it may hamper, from the other, the use of PD-L1/2 expression on tumor cells as a predictive biomarker for response.

The therapeutic use of PD-1 inhibitors, nivolumab and pembrolizumab, in patients with relapsed and refractory (RR) HL has been initially explored in two early-phase studies. In the expansion cohort of the phase Ib trial (CA209-039), 23 patients with heavily pretreated RR$\mathrm{HL}$ were given nivolumab $3 \mathrm{mg} / \mathrm{kg}$ every two weeks until response, tumor progression or intolerable side-effects [56]. Of the enrolled patients, $78 \%$ had progressed after autologous stem cell transplantation (ASCT) and $78 \%$ had failed on brentuximab vedotin (BV). ORR was $87 \%$ with a complete response rate of $22 \%$. At a median follow up of 101 weeks, the median DOR and median PFS were not yet reached [57]. Nivolumab was overall welltolerated with few grade 3/4 toxicities, mostly involving blood, skin, the gastrointestinal tract and lungs. Interestingly, in the one patient that was retreated with nivolumab post-progression, a second response was achieved. Analysis of pretreatment tumor specimens from 10 patients revealed copy-number gains and increased expression for

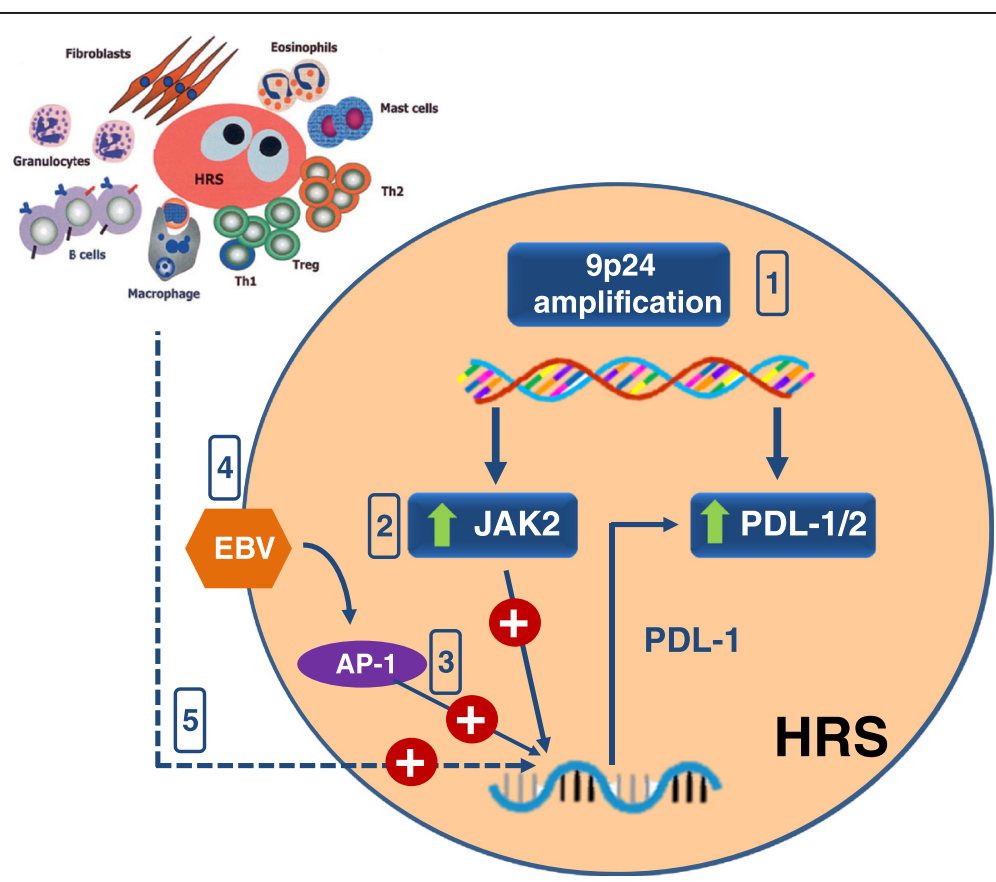

Fig. 3 Mechanisms leading to PD1 ligands expression in Hodgkin Reed-Sternberg (HRS) cells of Hodgkin Lymphoma. Multiple mechanism are concurrently implicated in overexpression of PD1-ligands by tumor cells of Hodgkin lymphoma. 1) Amplification of 9p24 increases gene dosage for PD-L1/2 and JAK2. 2) JAK2 gene product increases transcription of the PD-L1 gene. 3) HRS cells display the constitutive activation of AP1 whivh binds to an AP-1-responsive enhancer in the PD-L1 gene. 4) The Epstein-Barr Virus (EBV)-encoded latent membrane protein 1 increases PD-L1 promoter activity, also via AP-1. 5) The network underlying tumor microenvironment formation and maintenance in Hodgkin lymphoma results in the constitutive production of cytokines known to enhance activity of the PD-L1 gene 
both PD-L1 and PD-L2. Within the Checkmate 205 program, the CA209-205 study in RR-HL has recently completed the accrual for the original three cohorts, involving more than 250 patients, and a further cohort is being opened to test upfront treatment with single agent nivolumab followed by a nivolumab/AVD (doxorubicin, vinblastine, dacarbazine) combination for chemo-naïve patients with advanced $\mathrm{HL}$.

In the HL cohort of the KEYNOTE-013 study, 31 patients with RR-HL, who had progressed after BV and $67 \%$ of whom had also failed ASCT, received pembrolizumab $(10 \mathrm{mg} / \mathrm{kg}$ every 2 weeks) $[58,59]$. The ORR was $65 \%$ with a CR rate of $16 \%$. The median PFS at 24 weeks was $69 \%$ with a median DOR $\geq 24$ weeks in $71 \%$ of patients who achieved complete or partial response (range: $0.14-74+$ weeks). There were no AEs of grade higher than 3 and the most common treatmentrelated AEs were hypothyroidism (16 \%), diarrhea (13\%), nausea (13\%), and pneumonitis (10\%). Based on these impressive response rates and significant response duration, a number of further trials involving PD-1(e.g., nivolumab, pembrolizumab) and PD-L1- (durvalumab) targeted antibodies have been activated in patients with RR-HL. Furthermore, combinations of anti-PD1 antibodies with other immune checkpoints inhibitors such as ipilimumab and with BV are being currently tested in the same patient setting. The combination of PD-1 inhibitors with BV appears particularly intriguing since the sustained clinical response achieved with this latter agent, an anti-CD30 antibody conjugated with monomethyl-auristatin $\mathrm{E}$, a toxic poison of the dolastin family, have been related, beyond its direct cytotoxic activity, to the stimulation of an anticancer immune response [60]. In an immunocompromised murine model, therapeutic synergies were observed when combining dolastatins with tumor antigen-specific vaccination or PD-1/PD-L1 and CTLA-4 blockade [61]. This provides a strong rationale for treatment strategies that combine BV with immune-based therapies in patients with HL.

\section{Non-Hodgkin lymphoma}

In this group of lymphomas, the more heterogeneous pattern of PD-1 and its ligands on tumor cells may render more complex the interpretation of early clinical results obtained with some of the immune checkpoint inhibitors. One exception is represented by PMBCL, since in this aggressive NHL tumor cells often share the 9p24.1 amplification and constitutive PD-L1/2 overexpression typical of HL [54]. In this regard, the PMBCL cohort of KEYNOTE-013 study enrolled 10 heavily pretreated patients who also relapsed after or were ineligible for ASCT [62]. In nine evaluable patients, the ORR was $44 \%$, with one patient achieving a CR and three patients achieving a partial response. At a median follow-up of
144 days, the median DOR was not reached $(1+$ to $291+$ days), with all four responses ongoing at the time of data cutoff. These preliminary results fully justify further studies in this unique type of NHL.

Within the aggressive lymphomas, DLBCL represents the most common form accounting for about 30 to $35 \%$ of all NHL in the adults. Based on specific molecular signatures, two main biological subtypes with a different prognosis have been identified. In the GC-type of DLBCL, tumor cells rarely express PD-L1/2 or PD- 1 and the presence of $\mathrm{PD}-1+\mathrm{T}$ cells in the microenvironment is of unusual occurrence [53, 63]. Conversely, in DLBCL of ABC-type, neoplastic cells are characterized by a more sustained expression of PD-L1/2, but not of PD-1, and an excess of PD-1+ T cells can be found in the tumor microenvironment in some cases [53, 63]. In both CG- and ABC-DLBCL, however, variable amounts of PD-L1 and/or PD-1-expressing monocytes, histiocytes, dendritic cells and T/NK cells can be found in the microenvironment, supporting the presence of PD-1mediated interactions in the immune networks operating in these tumors [53].

In a first single-arm phase 2 study, 66 eligible patients with RR-DLBCL, were given pidilizumab $(1.5 \mathrm{mg} / \mathrm{kg}$ every 42 days) in the attempt to prevent early progression in those showing overt residual disease after ASCT and to consolidate response in those who achieved a complete response after transplantation [64]. Treatment yielded to a CR rate of $34 \%$ and ORR of $51 \%$ among patients with measurable disease after transplant. In the whole cohort of patients a 16-month PFS from first treatment of $72 \%$ was recorded along with an OS exceeding $80 \%$. These figures met the primary study endpoints and a concurrent, hypothesis-generating, immunoprofiling of circulating cells was consistent with an 'on-target 'in vivo effect of pidilizumab. No data were available as to relative frequencies of GC- versus ABC-DLBCL subtypes within the accrued patient population. Of note, it has recently been revealed that the target of pidilizumab is not, as was originally thought, PD-L1. Although its exact mechanism of action has not yet been explained, it has been stated that its administration is associated with enhanced maturation and survival of $\mathrm{T}$ lymphocytes, which may improve adaptive immunity, as well as activation of natural killer cells, which may improve innate immunity [65]. In the expansion cohort of the CA209-139 nivolumab study in lymphoid malignancies, 10 RR-DLBCL patients, failing after or ineligible for ASCT, were enrolled [66]. The ORR was $36 \%$ and a complete response was obtained in two patients, with a median overall DOR of 22 weeks.

FCL, beyond representing the second most common form of NHL in the Western world (22-25\% of all cases), may be of particular interest in the setting of immune checkpoint blockade strategies. Several molecular 
studies have confirmed that specific 'immune signatures', mirroring the presence and activity of several types of immune effectors in the lymphoma microenvironment, may strongly and independently predict prognosis in this indolent lymphoma [67]. In addition, while tumor cells do not usually express PD-L1 or PD-1, the FCL microenvironment is highly enriched for PD-1 and/or PD-L1/ 2 immune effectors [53]. In a single-arm phase 2 study, 29 eligible patients with RR-FCL, all previously exposed to rituximab, were treated with four courses of pidilizumab (3 mg/kg every 4 weeks) followed by eight monthly optional infusions in patients showing at least a stable disease [68]. After 17 days from the first infusion of pidilizumab, patients also received four weekly doses $\left(375 \mathrm{mg} / \mathrm{m}^{2}\right)$ of rituximab. The combination of pidilizumab plus rituximab was very well tolerated and devoid of grade 3 and 4 adverse events. Of the enrolled patients, 19 (66 \%) achieved an objective response, that was complete in 15 (52\%). The median PFS for all patients was of 18.8 months, and was not reached, at time of analysis, for the 19 patients in complete or partial response after pidilizumab plus rituximab. A concurrent immunoprofiling study, confirmed the 'on target' activity of the combination. The expansion cohort of the CA209-139 nivolumab study enrolled 10 patients with RR-FCL [66]. Four patients (40 \%) achieved an objective response, including a complete response and four partial responses. At a median follow up of 91 weeks, the median DOR for responding patients was not yet reached. The favorable toxicity profile of nivolumab was confirmed in these patients.

These extremely promising results are prompting an increasing number of trials targeted to specific NHL subtypes to better establish activity and mechanism of action of different immune checkpoint inhibiting-agents within such a heterogeneous group of tumors. Studies are also being launched to test newer combinations with antibodies targeting CD20 and other surface structures of tumor B cells and with molecules which target the known cell-signaling aberrations of the various NHL subtypes. The outstanding results obtained in patients with RR-HL led to the accelerated approval of nivolumab in the US and to its ongoing registration in Europe, where patient-named programs with this agent are currently ongoing. Studies testing the introduction of immune checkpoint inhibitors for the upfront treatment of HL have already been launched.

\section{Conclusions}

Treatment strategies that target the immune system provide the opportunity for antitumor activity across multiple cancer types, regardless of mutational status or tumor histology. While many of the initial advances in immunotherapy have been in melanoma, the focus has now broadened to include many other solid as well as hematological cancers. Different immunotherapeutic approaches are being evaluated across tumor types and their various novel mechanisms of action and safety profiles offer the potential for a variety of combination regimens. Ongoing and planned investigation of these immunotherapies, alone and in combination, represents the start of a new chapter in our treatment of cancer and offers the hope of better outcomes for patients with a wide range of cancers.

\section{Abbreviations}

$A B C$, activated $B$ cell; $A C T$, adoptive cell therapy; $A E$, adverse event; $A S C T$, authologous stem cell transplantation; BC, breast cancer; BV, brentuximab vedotin; $C A R$, chimeric antigen receptor; $C T L$, cytotoxic T lymphocyte; DCR, disease control rate; $\mathrm{DLBCL}$, diffuse large-cell $\mathrm{B}$ lymphoma; $\mathrm{DOR}$, duration of response; $\mathrm{EBV}$, EpsteinBarr virus; FCL, follicular cell lymphoma; FDA, Food and Drug Administration; GC, germinal-center; Gl, gastrointestinal; HCC, hepatocellular carcinoma; HL, Hodgkin lymphoma; HNSCC, head and neck squamous cell carcinoma; HPV, human papillomavirus; $\mathrm{HR}$, hazard ratio; IDFS, invasive disease-free survival; IDO, indoleamine 2,3-dioxygenase; IHC, immunohistochemistry; NHL, non-Hodgkin lymphoma; NPC, nasopharyngeal carcinoma; NSCLC, non-small-cell lung cancer; ORR, overall response rate; OS, overall survival; $\mathrm{PCR}$, pathological complete response; PD-1, programmed cell death protein 1; PD-L1, programmed death ligand 1; PFS, progression-free survival; $P M B C L$, primary mediastinal B cell lymphoma; PS, proportion score; RR, relapsed/refractory; TAA, tumor-associated antigen; TACE, transarterial chemoembolization; TIL, tumor-infiltrating lymphocyte; TNBC, triple-negative breast cancer; TUMAPS, tumor-associated peptides

\section{Acknowledgements}

Editorial assistance was provided by Andy Bond, and this manuscript was supported by S.C.I.T.O. - Campania Society of ImmunoTherapy of Cancer. A special thanks to Alessandra Trocino, for providing excellent bibliography service and assistance.

\section{Authors' contributions}

PAA, GC, CG, SP, AP, CC, LB, SPE, RM, VM, BD and SDP drafted and revised the manuscript critically for important intellectual content; all authors read and approved the final manuscript.

\section{Competing interests}

PAA has/had a consultant/advisory role for Bristol-Myers Squibb (BMS), Roche-Genentech, Merck Sharp \& Dohme (MSD), Ventana, Novartis, Amgen and Array; he received also research funds from Bristol Myers Squibb, RocheGenentech, Ventana, and Array. GC received research grants from Bayer, Novartis Pharma, Pfizer, Janssen, and Roche and has acted as a paid consultant or speaker for Eli Lilly, Italfarmaco, and Pfizer. CG has attended advisory boards and/or is speaker bureau member for BMS, MSD, Roche, AstraZeneca. SP has received honoraria from Roche, AstraZeneca, Pharmamar, and Pfizer. AP has received research support from Takeda Millennium and lecture fees and/or attended advisory boards for Roche, BMS, Takeda, Celgene, and Spectrum. BD has participated in an advisory board for BMS. SDP has attended advisory boards for GlaxoSmithKline (GSK), Novartis, Roche, Celgene, AstraZeneca, Amgen, Eisai, Italfarmaco, and Pfizer and has been an invited speaker for GSK, Novartis, Roche, Celgene, Astrazeneca, Amgen, and Teva. CC, LB, SPE, RM and VM have no competing interest to declare.

\section{Author details}

'Istituto Nazionale Tumori Fondazione G. Pascale - Unit of Melanoma, Cancer Immunotherapy and Innovative Therapy, Via Mariano Semmola, 80131 Naples, Italy. ${ }^{2}$ Dipartimento di Oncopneumoematologia, Unit of Medical Oncology, A.O.R.N. "A. Cardarelli", Naples, Italy. ${ }^{3}$ Division of Medical Oncology, "S. G. Moscati" Hospital, Avellino, Italy. ${ }^{4}$ Department of Urology and Gynecology, Istituto Nazionale Tumori Fondazione "G. Pascale", Via Mariano Semmola, 80131 Naples, Italy. ${ }^{5}$ Department of Hematology and Stem Cells Transplantation, Istituto Nazionale Tumori Fondazione "G. Pascale", Via Mariano Semmola, 80131 Naples, Italy. ${ }^{6}$ Division of Early Drug Development for Innovative Therapies, Istituto Europeo di Oncologia, Milan, 
Italy. ${ }^{7}$ Department Experimental Oncology, Lab of Molecular Biology \& Viral Oncology, Istituto Nazionale Tumori Fondazione "G. Pascale", Via Mariano Semmola, 80131 Naples, Italy. ${ }^{8}$ Department of Medicine and Surgery, University of Salerno, Baronissi, Salerno, Italy. ${ }^{9}$ Unit of Oncology, P.O. "Rizzoli" of Ischia, Ischia, NA, Italy. ${ }^{10}$ Unit of Oncology, A.O.R.N. dei COLLI "Ospedali Monaldi-Cotugno-CTO", Naples, Italy. " Department of Oncology, A.O. "G. Rummo", Benevento, Italy. ${ }^{12}$ Department of Molecular and Clinical Endocrinology and Oncology, University "Federico II", Naples, Italy.

\section{Received: 13 May 2016 Accepted: 30 June 2016}

\section{Published online: 19 July 2016}

\section{References}

1. Pagès F, Galon J, Dieu-Nosjean MC, Tartour E, Sautès-Fridman C. Fridman WH Immune infiltration in human tumors: a prognostic factor that should not be ignored. Oncogene. 2010;29:1093-102.

2. Ascierto ML, Melero I, Ascierto PA. Melanoma: from incurable beast to a curable bet. the success of immunotherapy. Front Oncol. 2015;5:152.

3. Wang $E$, Uccellini L, Marincola FM. A genetic inference on cancer immune responsiveness. Oncoimmunology. 2012;1:520-5.

4. Topalian SL, Drake CG, Pardoll DM. Immune checkpoint blockade: a common denominator approach to cancer therapy. Cancer Cell. 2015;13(27):450-61.

5. Garon EB, Rizvi NA, Hui R, Leighl N, Balmanoukian AS, Eder JP, et al. Pembrolizumab for the treatment of non-small-cell lung cancer. N Engl J Med. 2015;372:2018-28.

6. Herbst RS, Baas P, Kim DW, Felip E, Pérez-Gracia JL, Han JY, et al Pembrolizumab versus docetaxel for previously treated, PD-L1-positive, advanced non-small-cell lung cancer (KEYNOTE-010): a randomised controlled trial. Lancet. 2015;387:1540-50.

7. Gettinger SN, Horn L, Gandhi L, Spigel DR, Antonia SJ, Rizvi NA, et al. Overall survival and long-term safety of nivolumab (anti-programmed death 1 antibody, BMS-936558, ONO-4538) in patients with previously treated advanced non-small-cell lung cancer. J Clin Oncol. 2015;33:2004-12.

8. Rizvi NA, Mazières J, Planchard D, Stinchcombe TE, Dy GK, Antonia SJ, et al. Activity and safety of nivolumab, an anti-PD-1 immune checkpoint inhibitor, for patients with advanced, refractory squamous non-small-cell lung cancer (CheckMate 063): a phase 2, single-arm trial. Lancet Oncol. 2015;16:257-65.

9. Antonia SJ, Brahmer JR, Gettinger SN, Chow LQM, Juergens RA, Shepherd FA, et al. Nivolumab (anti-PD-1; BMS-936558, ONO-4538) in combination with platinum-based doublet chemotherapy (PT-DC) in advanced non-small cell lung cancer (NSCLC). J Clin Oncol. 2014;32(Suppl):8113.

10. Borghaei H, Paz-Ares L, Horn L, Spigel DR, Steins M, Ready NE, et al. Nivolumab versus docetaxel in advanced nonsquamous non-small-cell lung cancer. N Engl J Med. 2015;373:1627-39.

11. Brahmer J, Reckamp KL, Baas P, Crinò L, Eberhardt WE, Poddubskaya E, et al. Nivolumab versus docetaxel in advanced squamous-cell non-small-cell lung cancer. N Engl J Med. 2015;373:123-35.

12. Antonia SJ, Ou SI, Khleif S, Brahmer JR, Blake-Haskins A, Robbins PB, et al. Clinical activity and safety of MEDI4736, an anti-programmed cell deathligand 1 (PD-L1) antibody, in patients with non-small cell lung cancer. Ann Oncol. 2014;25(suppl_4):iv426-70.

13. Horn L, Spigel DR, Gettinger SN, Antonia SJ, Gordon MS, Herbst RS, et al. Clinical activity, safety and predictive biomarkers of the engineered antibody MPDL3280A (anti-PDL1) in non-small cell lung cancer (NSCLC): update from a phase la study. J Clin Oncol. 2015;33(Suppl):8029.

14. Spira Al, Park K, Mazières J, Vansteenkiste JF, Rittmeyer A, Ballinger M, et al. Efficacy, safety and predictive biomarker results from a randomized phase ॥ study comparing MPDL3280A vs docetaxel in 2 L/3L NSCLC (POPLAR). J Clin Oncol. 2015;33(Suppl):8010.

15. Aaltomaa S, Lipponen $\mathrm{P}$, Eskelinen M, Kosma VM, Marin S, Alhava E, Syrjänen K. Prognostic factors after 5 years follow-up in female breast cancer. Oncology. 1992;49:93-8.

16. Loi S, Michiels S, Lambrechts D, Fumagalli D, Claes B, Kellokumpu-Lehtinen PL, et al. Somatic mutation profiling and associations with prognosis and trastuzumab benefit in early breast cancer. J Natl Cancer Inst. 2013;105:960-7.

17. Loi S, Michiels S, Salgado R, Sirtaine N, Jose V, Fumagalli D, et al. Tumor infiltrating lymphocytes are prognostic in triple negative breast cancer and predictive for trastuzumab benefit in early breast cancer: results from the FinHER trial. Ann Oncol. 2014;25:1544-50.
18. Denkert C, Loibl S, Noske A, Roller M, Müller BM, Komor M, et al. Tumorassociated lymphocytes as an independent predictor of response to neoadjuvant chemotherapy in breast cancer. J Clin Oncol. 2010;28:105-13.

19. Denkert C, von Minckwitz G, Brase JC, Sinn BV, Gade S, Kronenwett R, et al. Tumor-infiltrating lymphocytes and response to neoadjuvant chemotherapy with or without carboplatin in human epidermal growth factor receptor 2-positive and triple-negative primary breast cancers. J Clin Oncol. 2015;33:983-91.

20. Loi S, Sirtaine N, Piette F, Salgado R, Viale G, Van Eenoo F, et al. Prognostic and predictive value of tumor-infiltrating lymphocytes in a phase III randomized adjuvant breast cancer trial in node-positive breast cancer comparing the addition of docetaxel to doxorubicin with doxorubicinbased chemotherapy: BIG 02-98. J Clin Oncol. 2013;31:860-7.

21. Salgado R, Denkert C, Campbell C, Savas P, Nucifero P, Aura C, et al. Tumorinfiltrating lymphocytes and associations with pathological complete response and event-free survival in HER2-positive early-stage breast cancer treated with lapatinib and trastuzumab: a secondary analysis of the NeoALTTO Trial. JAMA Oncol. 2015;1:448-54.

22. Dieci MV, Criscitiello C, Goubar A, Viale G, Conte P, Guarneri V, et al. Prognostic value of tumor-infiltrating lymphocytes on residual disease after primary chemotherapy for triple-negative breast cancer: a retrospective multicenter study. Ann Oncol. 2014;25:611-8.

23. Adams S, Gray RJ, Demaria S, Goldstein L, Perez EA, Shulman LN, et al. Prognostic value of tumor-infiltrating lymphocytes in triple-negative breast cancers from two phase III randomized adjuvant breast cancer trials: ECOG 2197 and ECOG 1199. J Clin Oncol. 2014;32:2959-66.

24. Loi S, Drubay D, Adams S, Francis PA, Joensuu H, Dieci MV, et al. Pooled individual patient data analysis of stromal tumor infiltrating lymphocytes in primary triple negative breast cancer treated with anthracycline-based chemotherapy. Presented at the 2015 San Antonio Breast Cancer Symposium. http://www.abstracts2view.com/sabcs15/view.php?nu=SABCS15L_857

25. Perez EA, Ballman KV, Anderson SK, Thompson EA, Badve SS, Bailey H, et al. Stromal tumor-infiltrating lymphocytes(S-TILs): In the alliance N9831 trial STILs are associated with chemotherapy benefit but not associated with trastuzumab benefit. Presented at the 2014 San Antonio Breast Cancer Symposium. http://www.abstracts2view.com/sabcs14/view. php?nu=SABCS13L_1455

26. Berek JS, Edwards RP, Parker LP, DeMars LR, Herzog TJ, Lentz SS, et al. Catumaxomab for the treatment of malignant ascites in patients with chemotherapy-refractory ovarian cancer: a phase II study. Int J Gynecol Cancer. 2014;24:1583-9.

27. Secord AA, Blessing JA, Armstrong DK, Rodgers WH, Miner Z, Barnes MN, et al. Phase II trial of cetuximab and carboplatin in relapsed platinum-sensitive ovarian cancer and evaluation of epidermal growth factor receptor expression: a Gynecologic Oncology Group study. Gynecol Oncol. 2008;108:493-9.

28. Steffensen KD, Waldstrøm M, Pallisgård N, Lund B, Bergfeldt K, Wihl J, et al. Panitumumab and pegylated liposomal doxorubicin in platinum-resistant epithelial ovarian cancer with KRAS wild-type: the PaLiDo study, a phase ॥ nonrandomized multicenter study. Int J Gynecol Cancer. 2013;23:73-80.

29. Hamanishi J, Mandai M, Ikeda T, Minami M, Kawaguchi A, Matsumura N, et al. Efficacy and safety of anti-PD-1 antibody (Nivolumab: BMS-936558, ONO4538) in patients with platinum-resistant ovarian cancer. J Clin Oncol. 2014; 32(Suppl):5511.

30. Hamanishi J, Mandai M, Ikeda T, Minami M, Kawaguchi A, Matsumura N, et al. Durable tumor remission in patients with platinum-resistant ovarian cancer receiving nivolumab. J Clin Oncol. 2015;33(Suppl):5570.

31. Varga A, Piha-Paul SA, Ott PA, Mehnert JM, Berton-Rigaud D, Johnson EA, et al. Antitumor activity and safety of pembrolizumab in patients (pts) with PD-L1 positive advanced ovarian cancer: Interim results from a phase $\mathrm{lb}$ study. J Clin Oncol. 2015;33(Suppl):5510.

32. Disis $M L$, Patel MR, Pant $S$, Infante JR, Lockhart AC, Kelly K, et al. Avelumab (MSB0010718C), an anti-PD-L1 antibody, in patients with previously treated, recurrent or refractory ovarian cancer: A phase $\mathrm{lb}$, open-label expansion trial. J Clin Oncol. 2015;33(Suppl):5509.

33. Gray HJ, Gargosky SE, CAN-003 Study Team. Progression-free survival in ovarian cancer patients in second remission with mucin-1 autologous dendritic cell therapy. J Clin Oncol. 2014;32(Suppl):5504.

34. Doi T, Piha-Paul SA, Jalal SA, Mai-Dang H, Yuan S, Koshiji M, et al. Pembrolizumab (MK-3475) for patients (pts) with advanced esophageal carcinoma: Preliminary results from KEYNOTE-028. J Clin Oncol. 2015;33(Suppl):4010. 
35. Bang Y-J, Chung H-C, Shankaran V, Geva R, Catenacci DVT, Gupta S, et al. Relationship between PD-L1 expression and clinical outcomes in patients with advanced gastric cancer treated with the anti-PD-1 monoclonal antibody pembrolizumab (MK-3475) in KEYNOTE-012. J Clin Oncol. 2015; 33(Suppl):4001.

36. El-Khoueiry AB, Melero I, Crocenzi TS, Welling TH, Yau TC, Yeo W, et al. Phase $1 /$ II safety and antitumor activity of nivolumab in patients with advanced hepatocellular carcinoma (HCC): CA209-040. J Clin Oncol. 2015; 33(Suppl), LBA101.

37. Butterfield LH, Ribas A, Meng WS, Dissette VB, Amarnani S, Vu HT, et al. T-cell responses to HLA-A*0201 immunodominant peptides derived from alpha-fetoprotein in patients with hepatocellular cancer. Clin Cancer Res. 2003:9(16 Pt 1):5902-8.

38. Bray SM, Vujanovic L, Butterfield LH. Dendritic cell-based vaccines positively impact natural killer and regulatory $T$ cells in hepatocellular carcinoma patients. Clin Dev Immunol. 2011;2011:249281.

39. Lee WC, Wang HC, Hung CF, Huang PF, Lia CR, Chen MF. Vaccination of advanced hepatocellular carcinoma patients with tumor lysate-pulsed dendritic cells: a clinical trial. J Immunother. 2005;28:496-504.

40. El Ansary M, Mogawer S, Elhamid SA, Alwakil S, Aboelkasem F, Sabaawy HE, et al. Immunotherapy by autologous dendritic cell vaccine in patients with advanced HCC. J Cancer Res Clin Oncol. 2013;139:39-48.

41. Buonaguro L, Petrizzo A, Tagliamonte M, Tornesello ML, Buonaguro FM. Challenges in cancer vaccine development for hepatocellular carcinoma. J Hepatol. 2013;59:897-903.

42. Buonaguro L, HEPAVAC Consortium. Developments in cancer vaccines for hepatocellular carcinoma. Cancer Immunol Immunother. 2016;65:93-9.

43. Seiwert TY, Haddad Rl, Gupta S, Mehra R, Tahara M, Berger R, et al. Antitumor activity and safety of pembrolizumab in patients (pts) with advanced squamous cell carcinoma of the head and neck (SCCHN): Preliminary results from KEYNOTE-012 expansion cohort. J Clin Oncol. 2015; 33(Suppl), LBA6008.

44. Fang W, Zhang J, Hong S, Zhan J, Chen N, Qin T, et al. EBV-driven LMP1 and IFN- $\gamma$ up-regulate PD-L1 in nasopharyngeal carcinoma: Implications for oncotargeted therapy. Oncotarget. 2014;5:12189-202.

45. Zhang J, Fang W, Qin T, Yang Y, Hong S, Liang W, et al. Co-expression of PD-1 and PD-L1 predicts poor outcome in nasopharyngeal carcinoma. Med Oncol. 2015:32:86

46. Hsu C, Lee SH, Ejadi S, Even C, Cohen R, Le C, et al. Antitumor activity and safety of pembrolizumab in patients with PD-L1-positive nasopharyngeal carcinoma: Interim results from a phase 1b study. Eur J Cancer. 2015; 51(Suppl S3):S558. Abstract 2801.

47. Segal NH, Ou S-HI, Balmanoukian AS, Fury MG, Massarelli E, Brahmer JR, et al. Safety and efficacy of MEDI4736, an anti-PD-L1 antibody, in patients from a squamous cell carcinoma of the head and neck (SCCHN) expansion cohort. J Clin Oncol. 2015;33(Suppl):3011.

48. Siu LL, Papadopoulos KP, Tsai FY-C, Hansen AR, Robbins PB, Li X, et al. Phase study to evaluate the safety and efficacy of MEDI4736 in combination with tremelimumab in patients with recurrent or metastatic (R/M) squamous cell carcinoma of the head and neck (SCCHN). J Clin Oncol. 2015;33(Suppl), TPS3090.

49. Bachireddy P, Burkhardt UE, Rajasagi M, Wu CJ. Haematological malignancies: at the forefront of immunotherapeutic innovation. Nat Rev Cancer. 2015;15:201-15.

50. Swerdlow SH, Campo E, Harris NL, Jaffe ES, Pileri SA, Stein H, et al. WHO Classification of Tumours of the Haematopoietic and Lymphoid Tissue. 4th ed. Lyon: IARC Press; 2008.

51. Scott DW, Gascoyne RD. The tumour microenvironment in B cell lymphomas. Nat Rev Cancer. 2014;14:517-34

52. Aldinucci D, Gloghini A, Pinto A, De Filippi R, Carbone A. The classical Hodgkin's lymphoma microenvironment and its role in promoting tumour growth and immune escape. J Pathol. 2010;221:248-63.

53. Hawkes EA, Grigg A, Chong G. Programmed cell death-1 inhibition in lymphoma. Lancet Oncol. 2015;16:e234-45.

54. Green MR, Monti S, Rodig SJ, Juszczynski P, Currie T, O'Donnell E, et al. Integrative analysis reveals selective 9p24.1 amplification, increased PD-1 ligand expression, and further induction via JAK2 in nodular sclerosing Hodgkin lymphoma and primary mediastinal large B-cell lymphoma. Blood. 2010;116:3268-77.

55. Green MR, Rodig S, Juszczynski P, Ouyang J, Sinha P, O'Donnell E, et al. Constitutive AP-1 activity and EBV infection induce PD-L1 in Hodgkin lymphomas and posttransplant lymphoproliferative disorders: implications for targeted therapy. Clin Cancer Res. 2012;18:1611-8.

56. Ansell SM, Lesokhin AM, Borrello I, Halwani A, Scott EC, Gutierrez M, et al. PD-1 blockade with nivolumab in relapsed or refractory Hodgkin's lymphoma. N Engl J Med. 2015;372:311-9.

57. Ansell S, Armand P, Timmerman JM, Shipp MA, Garelik MBB, Zhu L, et al. Nivolumab in patients with relapsed or refractory classical Hodgkin lymphoma: Clinical outcomes from extended follow-up of a phase 1 study (CA209-039). Orlando: Presented at the 57th American Society of Hematology (ASH) Annual Meeting; 2015. Abstract 583.

58. Moskowitz $\mathrm{CH}$, Ribrag V, Michot JM, Martinelli G, Zinzani PL, Gutierrez M, et al. PD-1 blocakde with the monoclonal antibody pembrolizumab (MK-3475) in patients with classical Hodgkin lymphoma after brentuximab vedotin failure: preliminary results from a phase lb study (KEYNOTE-013). Blood. 2014;124:290.

59. Armand P, Shipp MA, Ribrag V, Michot J-M, Zinzani PL, Gutierrez M, et al. PD-1 blockade with pembrolizumab in patients with classical Hodgkin lymphoma after brentuximab vedotin failure: Safety, efficacy, and biomarker assessment. Orlando: Presented at the 57th American Society of Hematology (ASH) Annual Meeting; 2015. Abstract 584.

60. Gopal AK, Chen R, Smith SE, Ansell SM, Rosenblatt JD, Savage KJ, et al. Durable remissions in a pivotal phase 2 study of brentuximab vedotin in relapsed or refractory Hodgkin lymphoma. Blood. 2015;125:1236-43.

61. Müller P, Martin K, Theurich S, Schreiner J, Savic S, Terszowski G, et al. Microtubule-depolymerizing agents used in antibody-drug conjugates induce antitumor immunity by stimulation of dendritic cells. Cancer Immunol Res. 2014;2:741-55.

62. Zinzani PL, Ribrag V, Moskowitz C, Michot J-M, Kuruvill J, Balakumaran A, et al. Phase $1 \mathrm{~b}$ Study of PD-1 blockade with pembrolizumab in patients with relapsed/refractory primary mediastinal large B-Cell lymphoma (PMBCL). Blood. 2015;126(23):Abstract 3986.

63. Chen BJ, Chapuy B, Ouyang J, Sun HH, Roemer MG, Xu ML, et al. PD-L1 expression is characteristic of a subset of aggressive B-cell lymphomas and virus-associated malignancies. Clin Cancer Res. 2013;19:3462-73.

64. Armand P, Nagler A, Weller EA, Devine SM, Avigan DE, Chen YB, et al. Disabling immune tolerance by programmed death-1 blockade with pidilizumab after autologous hematopoietic stem-cell transplantation for diff use large B-cell lymphoma: results of an international phase II trial. J Clin Oncol. 2013;31:4199-206.

65. Medivation press release, December 21, 2015. Medivation Initiates Pivotal Clinical Trial of MDV9300 in Diffuse Large B-Cell Lymphoma. http://investors. medivation.com/releasedetail.cfm?releaseid=947733 [[accessed June 2016]].

66. Armand P, Timmerman J, Lesokhin A, Halwani A, Millenson M, Schuster S, et al. Nivolumab in patients with relapsed or refractory lymphoid malignancies and classical Hodgkin lymphoma: updated safety and efficacy results of a phase 1 study (CA209-039). The 20th Congress of the European Hematology Association (EHA); 2015; Abstract \#S808.

67. Dave SS, Wright G, Tan B, Rosenwald A, Gascoyne RD, Chan WC, et al. Prediction of survival in follicular lymphoma based on molecular features of tumor-infi Itrating immune cells. N Engl J Med. 2004;351:2159-69.

68. Westin JR, Chu F, Zhang M, Fayad LE, Kwak LW, Fowler N, et al. Safety and activity of PD1 blockade by pidilizumab in combination with rituximab in patients with relapsed follicular lymphoma: a single group, open-label, phase 2 trial. Lancet Oncol. 2014;15:69-77.

\section{Submit your next manuscript to BioMed Central and we will help you at every step:}

- We accept pre-submission inquiries

- Our selector tool helps you to find the most relevant journal

- We provide round the clock customer support

- Convenient online submission

- Thorough peer review

- Inclusion in PubMed and all major indexing services

- Maximum visibility for your research

Submit your manuscript at www.biomedcentral.com/submit 